\title{
Magnitude and Determinants of Antenatal and Delivery Service Utilization in Arba Minch Town, South Ethiopia
}

\author{
Ewnetu Firdawek*, Dayan Aragu \\ Arba Minch University, College of Medicine and Health Sciences, Arba Minch, Ethiopia
}

Email address:

ewnetuliyew@gmail.com (E. Firdawek), daueunited@yahoo.com (D. Aragu)

\section{To cite this article:}

Ewnetu Firdawek, Dayan Aragu. Magnitude and Determinants of Antenatal and Delivery Service Utilization in Arba Minch Town, South Ethiopia. Science Journal of Public Health. Vol. 3, No. 3, 2015, pp. 339-343. doi: 10.11648/j.sjph.20150303.16

\begin{abstract}
Introduction: Maternal health care services utilization could save unnecessary severe complications and death among women during pregnancy, delivery and after delivery. Numerous factors such as characteristics of the women and their family, characteristics of illness as well as the health care system, including accessibility, acceptability, cost and quality of care, are contributed directly with the use of maternal health care. Thus it is necessary to examine these and other determinants of maternal health service utilization. Objectives: To assess the magnitude and determinants of Antenatal and delivery service utilization of pregnant women and women who delivered two years prior to the study in Arba Minch town. Methodology: Community based cross sectional study has been conducted on pregnant women and women who delivered two years prior to the study found in Arba Minch town in 2010. Simple random sampling technique was used to get the study subjects. Data were collected by trained personnel using a pre tested standardized questionnaire. Results: Among 774 respondents, mean number of pregnancy and delivery were 2.4 and 2.2 respectively. From mothers who were pregnant during the study period and 2 years prior to the study period, 715(92.4\%) had ANC checkup and 478(66.8\%) are preferred to deliver in health institutions. Level of education, marital status, husband's attitude toward ANC, planned pregnancy, illness experience during last and current pregnancy, perceived susceptibility to dangerous health problems were found significantly associated with ANC utilization. For delivery service in health institution, Level of education, husband attitude towards ANC, age at first pregnancy, plan for pregnancy and ANC checkup during pregnancy to be significant predictors. Conclusion: Level of education, age at first pregnancy, plan for pregnancy and husband's attitude were some of the factors that influence maternal health service utilization. Therefore programs and strategies should be focused on improving women education and male involvement in reproductive issue. More efforts also needed to strengthen the accessibility of maternal health services to the community.
\end{abstract}

Keywords: Antenatal Service Utilization, Delivery Service Utilization, Arba Minch Town

\section{Introduction}

Every day, 1500 women die from pregnancy or childbirth-related complications. In 2005, there were an estimated 536000 maternal deaths worldwide. Most of these deaths occurred in developing countries, and most were avoidable [1]. Improving maternal health is one of the eight Millennium Development Goals adopted by the international community at the United Nations Millennium Summit in 2000. In Millennium Development Goal 5 (MDG5), countries have committed to reducing the maternal mortality ratio by three quarters between 1990 and 2015. However, between 1990 and 2005 the maternal mortality ratio declined by only 5\%. Achieving Millennium Development Goal 5 requires accelerating progress [2].
A total of $99 \%$ of all maternal deaths occur in developing countries, where $85 \%$ of the population lives. More than half of these deaths occur in sub-Saharan Africa and one third in South Asia. The maternal mortality ratio in developing countries is 450 maternal deaths per 100000 live births versus 9 in developed countries [1]. Data show that less than two thirds $(62 \%)$ of women in developing countries receive assistance from a skilled health worker when giving birth. This means that 45 million home deliveries each year are not assisted by skilled health personnel [2].

In high-income countries, virtually all women have at least four antenatal care visits, are attended by a midwife and/or a doctor for childbirth and receive postnatal care. In low- and middle-income countries, just above two thirds of women get at least one antenatal care visit, but in some countries less than one third have this or, as in Ethiopia, just 12\% [3]. Even 
fewer women have the birth attended by a skilled health worker. The $63 \%$ average for low- and middle-income countries covers large differences: from $34 \%$ in eastern Africa to $93 \%$ in South America [4].

There are many reasons why women do not receive the care they need before, during and after childbirth. Many pregnant women do not get it because there are no services where they live, they cannot afford the services because they are too expensive or reaching them is too costly. Some women do not use services because they do not like how care is provided or because the health services are not delivering high-quality care. Further, cultural beliefs or a woman's low status in society can prevent a pregnant woman from getting the care she needs. To improve maternal health, gaps in the capacity and quality of health systems and barriers to accessing health services must be identified and tackled at all levels, down to the community [5].

In Ethiopia, the levels of maternal and infant mortality and morbidity are among the highest in the world. In 2005, the maternal mortality ratio was 720 per 100,000live birth \&there were 22,000maternal deaths in the same year. According to EDHS 2005, only $28 \%$ of women age $15-49$ were attended by a skilled health provider during their pregnancy $\&$ only $6 \%$ of the live births were attended by skilled birth attendant at delivery [6].

Despite the fact that maternal health care utilization is essential to reduce the deaths of mothers, little is known on the factors that affect utilization of these services in Arba Minch town. There for the objective of this study is to assess magnitude and factors associated with maternal health care services utilization \&came up with the possible recommendation for program planners who engage in the improvement of maternal \& child health care services

\section{Methodology}

Study setting \&period: The study was conducted in Arba Minch town from April 10- 25/2010. Arba Minch is located in the Southern part of Ethiopia which is $505 \mathrm{~km}$ away from Addis Ababa and the weather condition of the town is generally classified as hot. Currently there are 4 sub cities $\& 11$ kebeles(the smallest administrative unit) in the town. The projected population of the town in 2010 is 80,598 , from these $17,858(21.9 \%)$ of the population is women of child bearing age. There are two health centers \&one hospital in the town. The study will be conducted in four kebeles which are selected randomly. The study was conducted from April 10, 2010 up to April 25, 2010.

Study design: Community based cross sectional study was conducted.

Source population: All women who were pregnant \& gave birth two year prior to study and lived in A/Minch town.

Study population: Sampled women's of age 15-49 who were third trimester pregnant \&gave birth two year prior to study and lived in the selected kebeles for at least one year.

Sample size: A total of 774 study participants were obtained by using single population proportion formula by assuming the proportion of women's attending delivery care in the region $35.5 \%$ (6) with $5 \%$ of margin of error, $95 \%$ confidence level and $10 \%$ of non-response rate.

Sampling procedure: In Arba Minch there are 11 kebeles and each Kebeles were used as a cluster. From these 11 clusters, 4 clusters were selected by using simple random sampling technique and the required no of samples were taken from these selected kebeles. Then one house hold has been arbitrarily chosen, interview process continues until the required no of samples was obtained. Those households which haven't got an eligible mother for the interview were omitted.

Data collection procedure: A pre tested standardized interviewer administered questionnaire were used to collect the data. The questionnaire was adapted from other related studies \& modified according to the aim of the study. The questionnaire contains five parts including socio demographic characteristics of the respondents, questions related to ANC, respondent's knowledge on pregnancy and related health problems, choice of delivery \&assistance during delivery \& quality of antenatal care in respondent's perspective. The data was collected house to house by trained and experienced grade 12 completed and college students.

Data analysis: The collected data was entered into Epi info version 3.5.1 \& exported to SPSS version 17 computer software for analysis. Descriptive statistics like mean, median, percentage were used to describe study population in relation to different variables. Logistic regression analysis was also employed to assess the association between dependent \&independent variables. OR will be used to measure strength of association, \& p- value for statistical significance.

Ethical considerations: The study was first approved by ethical clearance committee of AMU. Letter of support was also written from research core process of Arba Minch University \& given to all concerned bodies. Informed consent was obtained from the participants prior to the interview and confidentiality was maintained.

\section{Results}

Socio Demographic characteristics of the respondents: All 774 proposed study participants were participated in the study making the response rate of $100 \%$. The age of the respondents ranged from 16 to 48 years and the mean age (SD) was $25.8 \pm 5.1$ years. Majority of the respondents 594 $(76.7 \%)$ were in the age range of $20-34$ years followed by 15-19 years $101(13.1 \%)$. In these two age categories, the percentage of ANC checkup and delivery at health institution were $554(77.5 \%)$ and $370(77.4 \%)$ and $92(12.9 \%)$ and 58 (12.1\%) respectively.

Concerning to level of education, majority 306 (39.5\%) completed their primary education followed by secondary school 276 (35.7\%). Most of the respondents 541(69.9\%) were housewives, followed by civil servants 57(7.4\%). Majority of the respondents $386(49.9 \%)$ were protestant by religion followed by orthodox $356(46 \%)$. Concerning the marital status almost all of them 704(91\%) were married. From those married respondents 693(97\%) had ANC 
checkup while they were pregnant and 458 (95.8\%) delivered in health institution.

The mean age of the mother at first pregnancy was $20.39 \pm 3.36$ with minimum age of $12 \&$ maximum age of 33 years. The mean number of pregnancy was $2.41 \pm 1.7$ with minimum of 7 and maximum of 11 pregnancies whereas the mean number of delivery was $2.18 \pm 1.68$ with minimum of 0 and maximum of 10 deliveries. Still birth was reported by $21(2.7 \%)$ of respondents and from these $20(1 \%)$ and 14 $(2.9 \%)$ of the respondents were attended ANC checkup and delivered in health institution respectively. About 36(4.7\%) of the respondents reported there was infant death. Majority of the respondents 706 (91.2\%) didn't face any abortion.

Antenatal follow up of the respondents: The mean number of family size who lived in the household was $4.49 \pm 1.88$ with minimum family size of 1 and maximum family size of 12. .Majority of the respondents $616(79.6 \%)$ were planned their current pregnancy while the rest 158(20.6) were unplanned. From those unplanned pregnancies 128 (17.9\%) respondents utilize ANC service and 72 (15.1\%) delivered in health institution. Majority of the respondents $721(93.2 \%)$ think that pregnant women could get ANC from health institutions. With regard to the source of information about ANC services, almost half of the respondents 338(43.7\%) heard from health institutions followed by relatives $237(30.6 \%)$. Concerning to the benefit of ANC, majority of the respondents $513(66.3 \%)$ said the benefit is for both mother \&child whereas $121(15.6 \%)$ for the mother only and $93(12 \%)$ for the child only. Majority of the respondents 715(92.4\%) had ANC checkup while the rest 59(7.6\%) didn't have any ANC checkup. Among those who had ANC checkup, $450(58.1 \%)$ of them start ANC checkup at the gestational age of 4-6 month and $187(24.2 \%)$ at 1-3 month. Of those who had ANC checkup, 343(44.3\%) had four and above ANC visit and 248(32\%) of them had three visit. With regard to the health institution that the respondents used for ANC checkup, 512(66.1\%) used health center and $199(25.7 \%)$ used hospital. The reason mentioned by the respondents why they used these health institutions were closeness of the health institution for 398(51.4\%), convince of the time for the service for $186(24 \%)$ and high quality of service for $153(19.8 \%)$.

With regard to TT vaccination during ANC visit, majority $695(97.2 \%)$ of them were vaccinated. Of those who were vaccinated, $578(83.16 \%)$ were vaccinated two times/above and $117(16.83 \%)$ of them only once. Almost half of them $413(59.4 \%)$ had vaccination card for TT which has been seen by the data collectors $\& 68(9.8 \%)$ of them didn't have vaccination card.

With regard to health education during ANC visit, $452(63.2 \%)$ of respondents said health education was given during the visits. Among those respondents 210(29.4\%) of them took health education on ANC and 163(22.8\%) on family planning. Concerning to blood pressure and weight measurement during ANC visit, almost all of the respondents $697(97.5 \%)$ and 684(95.7\%) were measured their BP and weight during ANC visit respectively. Laboratory examination like blood, urine, stool were carried out for $638(89.2 \%)$ of the respondents. Majority of the respondents $534(74.7 \%)$ were paid for ANC services. From those respondents who pay for the service, the payment was fair for $316(59.2 \%)$ of them.

The main reasons mentioned for not attending ANC checkup were too busy to attend ANC 12(20.3\%) followed by being in state of good health $10(17 \%)$.

Knowledge about ANC and pregnancy related health problems: Majority of respondents $755(97.5 \%)$ were agreed that a pregnant woman should attend ANC checkup. Among the respondents who were agreed, 387(51.3\%) of them mentioned gestational age/month to start ANC was 4-6 month followed by $1-3$ month for $336(44.5 \%)$. About $370(47.8 \%)$ of the respondents were aware about the danger signs related to pregnancy. Some of the danger signs mentioned by the respondents include persistent vomiting $136(36.8 \%)$, vaginal bleeding $111(30 \%)$ and anemia $101(27.3 \%)$. About a total of $253(32.7 \%)$ ) of respondents experience health problems while they were pregnant. From these respondents 249 (34.8\%) attended ANC checkup and $175(36.6 \%)$ delivered in health institutions.

Among many socio-demographic variables, level of education, marital status, husband's attitude toward ANC and family size were found to be associated with maternal health service utilization. Respondents with educational level of secondary education and above $[\mathrm{OR}=3.32,(95 \% \mathrm{CI}: 1.21$, 9.06)] were more likely to utilize ANC service than those who were not attended an education. Those respondents who were not married $[\mathrm{OR}=0.27,(95 \% \mathrm{CI}: 0.09,0.76)]$ were less likely to utilize ANC service than those who were married. Positive attitude of the husband towards ANC [OR=14.51, (95\% CI: $6.80,30.95)$ ] and those with 3 to 4 family size $[\mathrm{OR}=3.39,(95 \% \mathrm{CI}: 1.33,8.66)]$ were more likely to utilize the service than those husbands who had negative attitude towards ANC and those with 2 or less family size respectively. Among the obstetric factors, Respondents who plan their pregnancy $[\mathrm{OR}=4.07,(95 \% \mathrm{CI}: 2.28,7.28)]$, experience illnesses for their current or last pregnancy [OR= 5.34, (95\% CI: 1.84, 15.49)] and had perceived susceptibility to dangerous health problems $[\mathrm{OR}=4.05$, (95\% CI: 1.18 , 13.8)] were more likely to utilize ANC service than their counter parts.

Choices of delivery place and assistances during delivery: With regard to the place of delivery, 293(37.9\%) of delivered at home, 270(34.9\%) at hospital and 193(24.9\%), at health centers. The major reasons for choosing health institution for delivery were the quality of the service $234(50.3 \%$ ) followed by closeness of the institutions $141(30.3 \%)$. On the other hand the reasons for those respondents who delivered at home were sudden contractions 163(55.6\%) and unaffordability of the service in health institution $85(29 \%)$. Form those who delivered at home, 150(51.2\%) have got assistance from TBAs and 78(26.6\%) from neighbors.

Among socio demographic and obstetric variables, level of education, husband attitude towards $\mathrm{ANC}$, age at first pregnancy, planned pregnancy and ANC checkup during 
pregnancy to be significant predictors for utilizing delivery service in health institution. Respondents with educational level of secondary and above $[\mathrm{OR}=1.94$, (95\% CI: 1.23 , 3.06)] and positive attitude of husbands towards ANC $[\mathrm{OR}=5.75,(95 \% \mathrm{CI}: 2.73,12.12)]$ were more likely to deliver in health institution than those who were not attended an education and who had negative attitude respectively. Respondents whose age were above 18 at first pregnancy $[\mathrm{OR}=1.53,(95 \% \mathrm{CI}: 1.11,2.12)]$ were more likely to deliver in health institution than those with age were less than 18 at first pregnancy. Respondents who were pregnant without plan $[\mathrm{OR}=0.58,(95 \% \mathrm{CI}: 0.39,0.85)]$ were less likely to deliver in health institution than those with planned pregnancy. Respondents who had ANC checkup during their pregnancy $[\mathrm{OR}=5.43,(95 \% \mathrm{CI}: 2.75,10.72)]$ were more likely to deliver in health institution than their counter parts.

\section{Discussions}

The study explored several factors which determine utilization of maternal health service in Arba Minch town south Ethiopia. About 92.4\% of the respondents had ANC checkup while they were pregnant which is consistent with a survey in Kenya, republic of Congo and study in Hadiya zone Ethiopia [7, 8, and 9]

At least two TT vaccinations were reported by $83.2 \%$ of ANC care attendees which is higher than the finding in Afar region and Hadiya zone, Ethiopia $[9,10]$. This could be due to time difference between two studies.

Seventy five percent of respondents have been paying for ANC service and for $59.2 \%$ of them the payment was fair which is consistent with findings in afar region Ethiopia [10].

Health institution delivery was $61.7 \%$ which is lower than

study in Burkina Faso and Kenya [7, 11]. This could be due the availability and accessibility of the health institution and knowledge gap between these two study populations.

Level of education had significant association with maternal health service utilization. Respondents with level of education above secondary school were more likely to utilize the service than their counter parts. Respondents in low level of education might not have enough knowledge about maternal health services and health problems related to pregnancy as result they might not use maternal health services.

Respondents who planned their pregnancy were more likely to utilize the service than those who had no plan. This might indicate the importance of planning and preparation for pregnancy has a paramount importance for utilization of maternal health services.

Positive attitude of husbands towards maternal health services was significantly associated with the utilization which is consistent with study in Afar region [10]. Thus involving male partners in all reproductive issue is important to encourage their partners to utilize the service.

In general, level of education and attitude of the husband are associated with maternal health service utilization, increasing educational opportunity for women's and promotions of behavioral change communication to change the husbands attitude will have a great contribution for maternal health service utilization. As age at first pregnancy above 18 was found to be good for utilization of maternal health service utilizations, policies should advocate on prohibiting early marriage and pregnancy is important.

\section{Tables}

\begin{tabular}{lllll}
\hline Variables & $\begin{array}{l}\text { ANC check up } \\
\text { Yes Number (\%) }\end{array}$ & No Number (\%) & Crude OR (95\%CI) & Adjusted OR (95\% CI) \\
$\begin{array}{l}\text { Level of education } \\
\text { Never attended }\end{array}$ & $167(23.4)$ & $25(42.4)$ & 1 & 1.00 \\
Elementary & $280(39.1)$ & $26(44.1)$ & $1.61(0.90-2.88)$ & $1.261(0.62-2.56)$ \\
Secondary \&above & $268(37.5)$ & $8(13.5)$ & $5.01(2.21-11.37)$ & $3.317(1.21-9.06)$ \\
Marital status & $693(97)$ & $48(81.4)$ & 1 & 1.00 \\
Married Never married & $21(3)$ & $11(18.6)$ & $0.13(0.06-0.28)$ & $0.27(0.09-0.76)$ \\
Husband attitude toward ANC & & & & $14.511(6.80-30.95)$ \\
Positive & $670(93.7)$ & $27(45.8)$ & $18.61(9.39-36.89)$ & $1.36(0.46-3.67)$ \\
Negative & $17(2.4)$ & $11(18.6)$ & $1.16(0.45-2.99)$ & 1 \\
Don't know & $28(3.9)$ & $21(35.6)$ & 1 & \\
\hline
\end{tabular}

\section{Acknowledgements}

We thank Arba Minch University for sponsoring this research. Our acknowledgment also goes to Arba Minch health bureau for providing information and materials for this study. Finally, we would like to acknowledge the study team, study participants and all others participated in this study.

\section{References}

[1] WHO, UNICEF, UNFPA and the World Bank. Maternal mortality in 2005: developed by WHO, UNICEF, UNFPA and the World Bank. Geneva; 2007. 
[2] Tracking progress in maternal, newborn \& child survival: the 2008 report. New York, United Nations Children's Fund; 2008. Web http://www.countdown2015mnch.org/index.php?option=com content $\&$ view $=$ article $\&$ id $=68 \&$ itemid $=61$.

[3] WHO and UNICEF. Antenatal care in developing countries: promises, achievements and missed opportunities. WHO/UNICEF; 2003.Web site: http://www.who.int/reproductive_health/global_monitoring/da ta.html. Countdown to 2015.

[4] World Health Organization. Proportion of births attended by a skilled health worker 2008 updates.WHO; Geneva: 2008.

[5] Maternal, newborn and child survival. 2008. Web site: http://www.countdown2015mnch.org/documents/.../ethiopia_2 0080304.pdf.

[6] Central Statistics Agency [Ethiopia] and ORC Macro. Ethiopia Demographic and Health survey 2005. Addis Ababa, Ethiopia and Calverton, Maryland, USA: Central Statistics Agency and ORC Macro; 2006.

[7] Rhoune Ochako, Jean-Christophe Fotso, Lawrence Ikamari, Anne Khasakhala. Utilization of maternal health services among young women in Kenya: Insights from the Kenya
Demographic and Health Survey. BMC Pregnancy Childbirth 2011; 11(1). doi: 10.1186/1471-2393-11-1.[BMC].

[8] Abel Ntambue ML, Francoise Malonga K, Michele Dramaix-Wilmet, Philippe Donnen. Determinants of maternal health services utilization in urban settings of the study of Lubumbashi city. BMC Pregnancy Childbirth 2012; 12(66). doi: 10.1186/1471-2393-12-66. [BMC].

[9] Zeine Abonsse, Mirkuzie Woldie, Shimeles Ololo. Factors Influencing Antenatal Care Service Utilization in Hadiya Zone. Ethiopian Journal of Health Science 2010; 20(2): 75-82.

[10] Fenta M. Assessment of factors affecting utilization of maternal health care services in ayssaita and dubti towns. A thesis presented to school of graduate studies Addis Ababa University 2005.

[11] De Allegri M, et al. Determinants of utilization of maternal care services after the reduction of user fees: A case study from rural Burkina Faso. Health Policy 2010, doi:10.1016/j.healthpol.2010.10.010. 